\title{
LA ACCIÓN POLÍTICA COMO HERRAMIENTA METODOLÓGICA EN IMPLEMENTACIÓN DE PROPUESTAS: UN CASO DE INVESTIGACIÓN CRÍTICO-PARTICIPATIVA
}

\author{
Andrés Godínez Mora* - Henry Pescod Vargas ${ }^{* *}$ - Esteban León Carballo***
}

Fecha de recepción: 30/01/2017

Fecha de aceptación: 18/04/2017

Resumen: El presente artículo presenta los aspectos teóricos que originaron la herramienta metodológica llamada "acción política" para investigaciones que requieran cambios en la cotidianeidad de los participantes del proyecto y su contexto. La investigación de este estudio está basaba en la construcción de cultura política a partir del uso de series de anime, además de promover un pensamiento crítico que se vea reflejado en obras de dibujo manga. Según lo anterior, se presentó la elaboración de propuestas para concientizar sobre problemáticas a partir de una metodología participativa grupal. Los principales hallazgos demostraron la viabilidad de proyectos de esta índole y la aplicabilidad en otros tipos de investigación y temáticas. Igualmente, esto permitió observar la aplicación de conocimientos adquiridos a partir de experiencias previas y el desarrollo de métodos para aplicarlo.

Palabras clave: metodología, cultura política, percepción, pensamiento crítico, enseñanza, Costa Rica.

\begin{abstract}
The following paper summarizes the theoretical aspects that originated the methodological tool called "political action" for researches that requires changes in the daily lives of the participants of the project and their context. The base research of this paper was based on the construction of political culture using anime series, besides it sought to promote critical thinking that would be reflected in works of manga drawing. According to the above, the development of proposals to raise awareness about different problems from a group participatory methodology. The main findings demonstrated the viability of such projects and the applicability in other types of research and topics. In addition, this allowed to observe the application of knowledge gained from previous experiences and the development of methods to apply this.
\end{abstract}

Keywords: methodology, political culture, perception, critical thinking, teaching, Costa Rica.

\footnotetext{
* Costarricense. Licenciado en Enseñanza de los Estudios Sociales y la Educación Cívica por la Universidad Nacional, Costa Rica (UNA). Trabaja en el Ministerio de Educación de Costa Rica (MEP). Correo electrónico: angod92@gmail.com

** Costarricense. Licenciado en Enseñanza de los Estudios Sociales y la Educación Cívica por la Universidad Nacional, Costa Rica (UNA). Trabaja en el Ministerio de Educación Pública de Costa Rica (MEP). Correo electrónico: henry_2000@hotmail.com

*** Costarricense. Licenciado en Enseñanza de los Estudios Sociales y la Educación Cívica por la Universidad Nacional, Costa Rica (UNA). Correo electrónico: esteban.leon.carballo@gmail.com
} 


\section{Introducción $^{1}$}

Este artículo se deriva de la investigación llamada "Anime y manga como medios de construcción de cultura política. Una experiencia con un grupo de estudiantes del Liceo Samuel Sáenz Flores”. Dicho estudio pretendió analizar cómo se manifiesta el anime ${ }^{2}$ y manga $^{3}$ en la cultura política de ese grupo estudiantil, con el fin de promover el pensamiento crítico. Las apreciaciones de los contenidos que proyectan estas series se dan acorde con la vinculación de percepciones del espectador; bajo estos preceptos, se consideró que el anime y manga en la cultura joven encuentra sus definiciones como un medio japonés que representa afinidades y aspectos culturales similares a los vistos en Occidente. .

La cultura política se analizó desde diferentes contextos, sujetos y temáticas. En especial destacó la importancia de haber incluido el ámbito privado como medio para generar consensos y disensos. Esto se da debido a que las percepciones permiten la generación de aprendizaje por medio del diálogo y crean espacios de expresión, es decir un mecanismo para generar cultura.

El pensamiento crítico también abarca gran variedad de temáticas, pero se enfoca principalmente en el cuestionamiento de la realidad y de los factores que afectan la conformación del entorno cercano. Este estado de conciencia permite que se manifieste la autodeterminación, codeterminación y solidaridad que lleva a las personas a ser autónomas y tomar acciones respecto al cambio de su entorno.

A manera general, el anime y manga como medios de comunicación necesitan visiones críticas para su estudio. Por lo tanto, cultura política y las teorías críticas de la educación se complementaron como mecanismo de análisis y como generadores de

\footnotetext{
${ }^{1}$ Este artículo está basado en un trabajo final de graduación para optar por el grado de licenciatura titulado, "Anime y manga como medios de construcción de cultura política. Una experiencia con un grupo de estudiantes del Liceo Samuel Sáenz Flores”, el cual fue elaborado en el marco del seminario de graduación "Los Estudios Sociales y la Cívica: sus interrelaciones con la sociedad".

2 "Anime" es el nombre que se suele utilizar para nombrar a las series animadas japonesas.

3 "Manga" es el nombre del estilo utilizado en las historietas o comics japoneses.
} 
acciones, el primero de tipo político y el segundo de tipo transformador, lo cual puede combinarse para generar propuestas acordes al aprendizaje de los estudiantes.

Por otro lado y, como parte del cuarto objetivo de ese trabajo investigativo, se elaboró una presentación de los productos -dibujos manga- generados por los estudiantes en la comunidad educativa, con el fin de discutir acerca del anime y manga y sus trasfondos políticos. En este último aspecto, se incluyeron los estudios del concepto de acción, en especial los realizados por la autora Hannah Arendt, como medio para cumplir con los fines esperados.

A partir de lo expuesto, la acción deriva de una práctica con libertad y espontaneidad tiene un inicio, es decir una razón de ser y por tanto, parte de la autonomía del ser humano para actuar y ejercer una práctica en el mundo. Ante esto, se señala que la acción tiene un fin impredecible, el cual se encuentra en la capacidad de la persona y en los alcances y limitaciones de su contexto (Arendt, 1997).

Cuando se realizó el trabajo investigativo que antecede a este artículo, se pensó en articular los estudios de la acción con las dimensiones "política" y "transformadora" que se pretendían utilizar como base para esa investigación. Por consiguiente, se reflexionó que una acción como tal está formada por un conjunto de prácticas que se realizan a lo largo de la cotidianidad, las cuales si bien no son relevantes en su mayoría, se pueden trasformar en acciones más profundas en algún ámbito específico, sobre todo en relación con la política, Arendt (1997) señala que:

La acción [...] sólo es política si va acompañada de la palabra -lexis.-, del discurso. Y ello porque, en la medida en que siempre percibimos el mundo desde la distinta posición que ocupamos en él, sólo podemos experimentarlo como mundo común en el habla. Sólo hablando es posible comprender, desde todas las posiciones, cómo es realmente el mundo. El mundo es pues lo que está entre nosotros, lo que nos separa y nos une (pp. 18-19).

A partir de lo dicho por Arendt, se revela que el diálogo es clave para el desarrollo de una acción política. Lo anterior señala que esta concepción sobre lo político traspasa las ideas tradicionales que lo enmarcan dentro de cuestiones electorales y de gobierno. Por otro 
lado, para que una práctica se convierta en acción política necesita de cuatro preceptos, los cuales son: la convicción -motivo-, el fin que persigue, la meta a que se orienta y el sentido que manifiesta al ejecutarse (Arendt, 1997).

Primeramente la convicción o el motivo se centra en un trasfondo relevante que se desea cambiar, es la evidencia de que el ejecutante puede haber percibido algún factor que imposibilita el desarrollo de algún proyecto o caso en específico, lo que a su vez denota cierta criticidad para dar el primer paso e intentar el cambio. Como otro aspecto, el fin que se persigue tiende a ser un factor relativo según los intereses del sujeto, donde si bien el cambio que se busca es positivo o negativo, dependerá de la visión política y su beneficio con las distintas partes que están envueltas en el proceso. Para diferenciar el fin y el sentido, Arendt (1997) explica que:

El sentido de una cosa, a diferencia del fin, está siempre encerrado en ella misma y el sentido de una actividad sólo puede mantenerse mientras dure esta actividad. Esto es válido para todas las actividades, también para la acción, persigan o no un fin. Con el fin de algo ocurre precisamente lo contrario; sólo hace su aparición en la realidad cuando la actividad que la creó ha llegado a su término (p. 125).

Con el fin solo se logrará visualizar que los actos incluidos en la acción política logran determinar un resultado, sea positivo o no, se comprobará si se ha alcanzado el propósito. Por otra parte, el sentido que se manifiesta tiende a ser la congruencia y mensaje que se quiere dar a entender junto con la acción política, es decir que si se promueven ideas de paz, el camino a tomar no es la violencia sino que es totalmente lo opuesto. De la misma manera, este sentido manifiesto es el paso visible de la acción y permite la anexión de simpatizantes con el motivo y la causa.

Respecto a la meta que se orienta, Arendt (1997) menciona que "son los [aspectos] conforme a los que debe juzgarse todo lo que se hace; sobrepasan o transcienden el acto en el mismo sentido en que toda medida transciende aquello que tiene que medir" (pp. 125126). Por tanto, se pueden definir los pasos a seguir para llevar a cabo el fin, juzgan la 
acción y, por consiguiente, se puede considerar como el plan de acción necesario para lograr este cambio.

Los aspectos señalados anteriormente generan un entorno que permite medir el proceso que transcurre entre el inicio de una acción y el final. En consecuencia, se evidenció la posibilidad de formular una metodología con base aen estos principios. Para ello la observación sería clave para poder determinar los resultados que determinen la relación entre el principio y los resultados de una acción política.

Respecto a la dimensión "transformadora", ésta se orientó hacia los principios de Wolgand Klafki (1986). Este autor también señala algunos aspectos en los cuales el pensamiento crítico puede lograr una transformación. Klafki (1986) señala que se necesitan tres elementos, "el interés por la acción, la configuración y el cambio" (p. 42). En otra perspectiva, el interés trata de la primera etapa mediada por la discusión o diálogo de los problemas entre los participantes de una acción transformadora. Consecuentemente, la configuración consiste en la planificación de la propuesta de cambio y, finalmente, el cambio es representado por la acción ejecutada para modificar una determinada problemática socio-política.

En resumen, utilizando los argumentos de Klafki (1986) y Arendt (1997), los pasos para una acción política transformadora son un interés para la acción que surge de la convicción o motivo de una persona o grupo. Posteriormente, se da la configuración que con base en un fin que se persigue, busque la construcción de una meta para orientar la acción y darle sentido. Como paso final, los resultados de este proceso visualizan el cambio y revelan el cumplimiento o no del fin.

La transformación tiende a estar abierta a todos los aspectos que englobe la política como tal, por lo que no es necesario un cambio radical en el sistema de gobierno o electoral para poder llevar a cabo un reajuste significativo a las distintas estructuras que se encuentran bajo este ámbito. En contraposición, una simple puesta en común en un sistema educativo puede alcanzar un proceso político mucho más complejo siguiendo la misma línea de acción política trasformadora con la que inició, dejando en claro el hecho de que poseen la misma estructura. 
La acción política como herramienta metodológica en implementación de propuestas: un caso de investigación crítico-participativa

Por último, la acción política transformadora es una herramienta que permite efectuar cambios en el contexto inmediato. A través de la elaboración de propuestas congruentes, se pueden llevar acabo planes de acción que promuevan los ideales de las personas o grupos que desean realizar esta transformación. Del mismo modo, busca que otros individuos se unan a los intereses que ellos siguen por un bien común.

\section{Metodología}

La acción política transformadora se trabajó con un grupo de 12 estudiantes, 10 hombres y 2 mujeres entre 14 y 17 años del Liceo Samuel Sáenz Flores. La elección de esta institución se dio debido a la relación que presenta con el anime. Al respecto, sus instalaciones se han encargado de albergar los festivales Kawaii y Kowai desde el 2008, los cuales consisten en un espacio interactivo para todos los fanáticos de este arte. Este evento permitió mostrar que el contexto y sus participantes de una u otra manera estaban envueltos por los diferentes tópicos que se querían estudiar -Cultura Política y Anime-.

Para fines informativos, los participantes fueron divididos en tres grupos denominados por los participantes como: Wasabi Shippudden, Ryōbi y Sagitario ${ }^{4}$. Dentro de Wasabi Shippudden, se encuentran cuatro hombres: Adrián, José, Alberto y Arturo. De igual manera, en Ryōbi están cuatro hombres: Antonio, Pedro, Ángel y John. Como último grupo, Sagitario presenta dos mujeres Ana y Angie y dos hombres Edgar y Flavio. Esta denominación se dio para mantener el anonimato de los menores de edad y para formar grupos focales.

Con respecto a la herramienta analizada en este artículo científico, tenía como fin elaborar dos presentaciones con la presencia de padres de familia, comunidad estudiantil y visitantes en las instalaciones del colegio. En dichos eventos, se presentaron los mangas realizados por los estudiantes y, a su vez, un diálogo sobre los propósitos que tiene la

\footnotetext{
${ }^{4}$ Estos nombres fueron elegidos por los participantes y son manifestaciones de sus gustos. Los dos primeros son referencias a la serie Naruto y el tercero a Saint Seiya-Caballeros del Zodiaco-.
}

Revista Perspectivas: Estudios Sociales y Educación Cívica - No. 14 ISSN-L: $2215-4728 \bullet$ enero-junio $2017 \bullet$ pp. 1-23 DOI: http://dx.doi.org/10.15359/rp.14.2 
visualización de este medio visual. Igualmente, dicho diálogo debía ser antecedido por una propuesta que planteaba cambiar la visión negativa del anime y manga.

Para este propósito, el pensamiento crítico debe manifestar en su deseo de transformación, el rompimiento de prejuicios y mitos promovidos por la tradición. En este sentido, el anime y manga presenta una percepción negativa a partir de posturas como el adultocentrismo, las autoridades religiosas, los medios de comunicación, entre otras. Al respecto, se esperaba romper con los mitos alrededor de la figura de estas series como por ejemplo su relevancia educativa, su contenido y sus efectos en los jóvenes. En consecuencia, se partió de una propuesta política y el énfasis al arte como una salida hacia la comunicación de problemas actuales y sus posibles soluciones.

Para analizar las dos presentaciones, se utilizó una observación participante que abarcó los diálogos entre los estudiantes, la recogida de artefactos y la observación de conversaciones casuales -informales- entre estudiantes y asistentes al evento. En este caso, los artefactos seleccionados fueron las propuestas de cambio que redactaron los estudiantes para su presentación.

El proceso para realizar estos eventos tuvo tres pasos: planificación, sistematización y ejecución. Dentro de la planificación, primeramente se buscó la fecha idónea en que existía una aglomeración de personas dentro del colegio. Como siguiente paso, se empezó un diálogo sobre los contenidos que debía tener la propuesta de cambio, se observó el interés por la acción, por la convicción o motivo de dicho interés y las posibilidades e impedimentos que valoraban los grupos que ejecutaban la acción.

Como segundo aspecto, la sistematización contenía la construcción de las propuestas de cambio con las ideas conversadas. Utilizando la recolección de artefactos -documentos escritos de las propuestas-, se observó la configuración del escrito que contenía el fin que se persigue y la meta a la que se orienta. Por último, la ejecución estaba representada por el cambio en sí que contenía el diálogo crítico con los asistentes, la capacidad resolutiva de conflictos -el alcance de consensos- y el sentido que se manifiesta 
al ejecutar la acción. En consecuencia, el mecanismo usado fue la observación de conversaciones casuales entre los participantes y los asistentes a la actividad.

Esta herramienta permitiría visualizar la construcción de las propuestas y su contenido, así como la interacción de los participantes con las personas que se acercaran a observar la presentación de las obras de manga. De igual manera, este proceso no afectó el desenvolvimiento de los jóvenes debido a que los investigadores están posicionados dentro de la rutina y no como un agente externo.

Como aspecto final, se estudiaron las propuestas de los tres grupos de estudiantes, las cuales estaban compuestas por su interés manifiesto y la configuración de esta idea. Posteriormente, se realizó una descripción sobre las presentaciones que fueron realizadas para exhibir las obras. Por último, se realizó un análisis de los eventos ocurridos a partir del diálogo crítico, la capacidad resolutiva de conflictos y el sentido que manifiestan al realizar la acción política transformadora.

\section{Reflexión e interpretación crítica}

En primer lugar, los participantes crearon tres historias manga, una por cada grupo. A partir de estas historietas se construyeron propuestas de cambio para romper con los mitos alrededor del anime y manga. Por lo tanto, cada obra tenía una problemática que abordar y un mensaje para los lectores. En consecuencia, al momento de la presentación, los estudiantes conversaban con los asistentes para llegar a acuerdos con respecto a la intención de sus trabajos.

De acuerdo al grupo llamado Wasabi Shippuden, ellos asentaron su propuesta en la historia que titularon como Kokoro Dream. El tema central de ésta fue la problemática de las obsesiones por el poder vistas desde diferentes ámbitos sociales -ver figura 1-. Al respecto, los estudiantes consideraban estas obsesiones como enfermedades, a lo que se aferra excesivamente o un vicio como elemento que no se puede dejar. 
Figura 1

Imagen sobre obsesiones en el manga "Kokoro Dream"
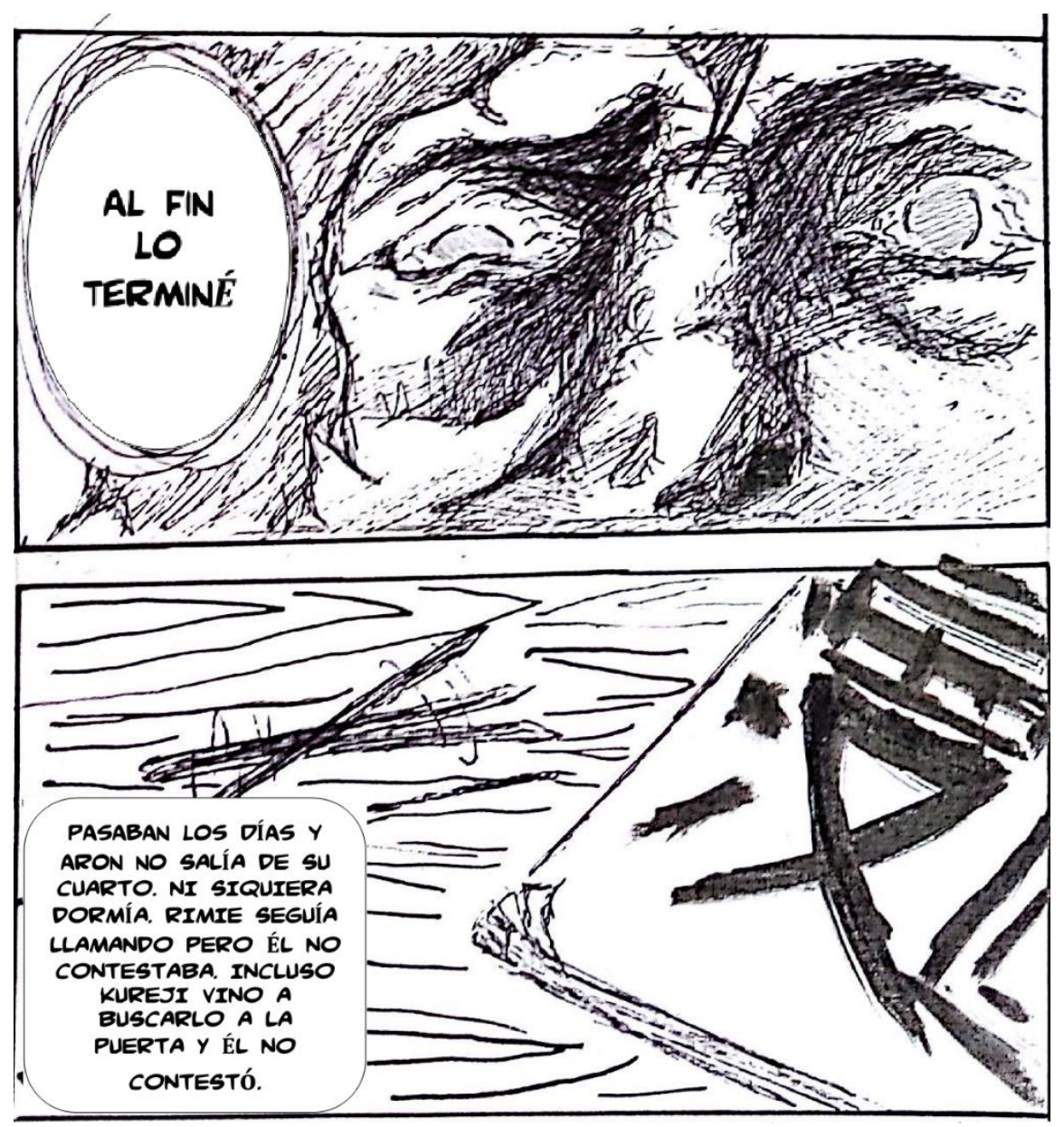

Fuente: Wasabi Shippuden, Kokoro Dream, diciembre, 2015.

Nota: Las obsesiones son una problemática que los autores quieren combatir por medio de la unión de personas para ayudar a la víctima a solucionar ese problema.

Respecto a los alcances de la propuesta de Wasabi Shippuden, los participantes esperaban servir de ejemplo para dar realismo a los eventos relacionados con las obsesiones. Además, buscaban ejemplificar un caso de afectación para funcionar como inspiración, en conjunto con un sentimiento de difusión para solidarizarse con estos casos. Estos aspectos partían de la concientización de las temáticas y la aceptación de los lectores. 
La acción política como herramienta metodológica en implementación de propuestas: un caso de investigación crítico-participativa

Otro elemento de esta idea fueron las debilidades que manifestaron los estudiantes, las cuales radicaban principalmente en la empatía por el tipo de obsesión. Este elemento se refiere a que el lector considere correcto lo realizado por los personajes. Por consiguiente, la persona no tomaría conciencia sobre lo expuesto sino que se puede sentir ofendido. De igual manera, los participantes temieron que los lectores no reconocieran la problemática del protagonista y no detectaran el mensaje que se quiso dar, así como el no abarcar todos los tipos de obsesiones.

El fin que persiguió esta propuesta, los estudiantes concordaron que se trataba de la reflexión sobre las consecuencias de las obsesiones y el reconocimiento de responsabilidades. Asimismo, la meta que se deseó era dejar lo habitual y elaborar una obra con un mensaje importante, abandonar el fanatismo y concentrarse en hacer una historia reflexiva. Igualmente, se desearon recalcar aspectos de la vida que son perdidos por esta problemática, por ejemplo la familia, los amigos y en sí toda socialización, incluyendo el daño físico y mental tras afrontar estos procesos.

Como reflexión de esta propuesta, los estudiantes desarrollaron los tres aspectos necesarios para el pensamiento crítico (Klafki, 1987). En primer lugar, buscaron la autodeterminación del lector al evitar las obsesiones y reaccionar en caso de afrontar una. Posteriormente, se codeterminaron para elaborar una historia que inspire a otros y permitiera asimilar el mensaje. Por último, se solidarizaron con las personas que afrontan estos casos al mostrar no solamente los aspectos negativos, sino la ayuda que necesitan para poder superarlas y recuperarse. Por lo tanto, se logró una acción política transformadora en sus primeras etapas.

Con respecto al grupo Sagitario, los colaboradores crearon una propuesta basada en su manga Spinne Himmel. Esta historia desarrolló su trama en un mundo, en el cual un grupo político dominante utiliza los medios de comunicación como un método de control masivo. Por consiguiente, la convicción o motivo que desarrollaron los estudiantes se basó en la desinformación de los medios de comunicación y su relación con el poder.

El grupo de informantes llamado Sagitario consideraba que sus alcances podían estar en las consecuencias que generan el creer todo lo que los medios comunican y no ser 
conscientes de la realidad. Por lo tanto, creían que esta historia podía colaborar con lo que llama el autor Apple (2008), el paso de una conciencia ingenua hacia un pensamiento crítico. De igual manera, se deseaba concientizar a los lectores sobre la importancia de la unión en casos en que una fuerza superior oprima los derechos.

En otro tema, las debilidades que encontraron los estudiantes de Sagitario se basaban principalmente en la creación de malentendidos. Lo anterior se debe a que la historia toma acción en un contexto de conflicto armado y por tanto, puede confundir el símbolo de la unión como un intento por llamar al armamentismo. De igual manera, no se deseaba que los lectores pensaran que los autores estaban en contra del sistema político, sino que existía una disconformidad con regímenes como el expuesto en Spinne Himmel -ver figura $2-$.

\section{Figura 2}

Imagen sobre manipulación de medios en el manga "Spinne Himmel"

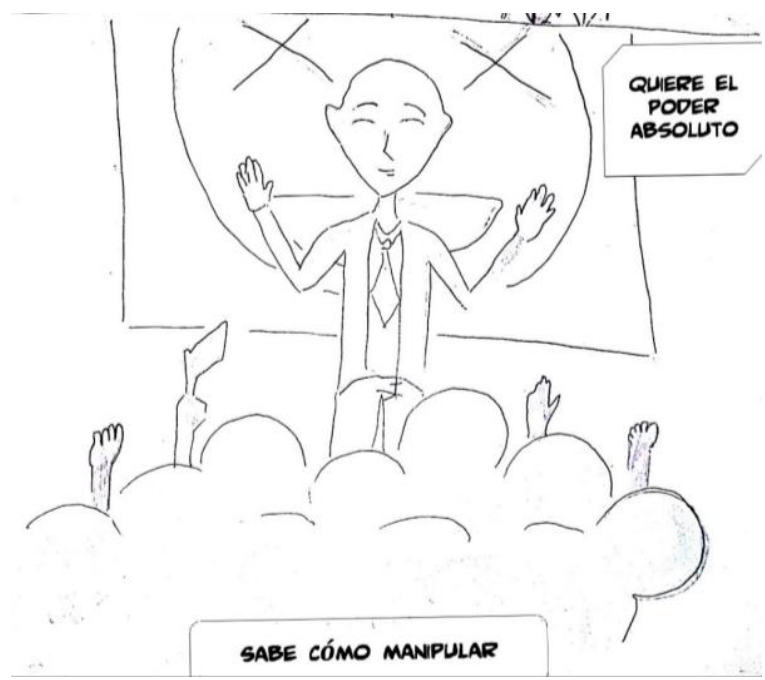

Fuente: Sagitario, Spinne Himmel, diciembre 2015.

Nota: Representación de la hegemonía por parte de los autores.

El fin que persiguió Sagitario con Spinne Himmel se orientó hacia la concientización de la lectura crítica de los medios de comunicación y la conformación de uniones en pro de derechos violados. Asimismo, intentaron romper el mito de que la hegemonía actual es un método $100 \%$ fiable e infalible, al representarlo como un sistema 
La acción política como herramienta metodológica en implementación de propuestas: un caso de investigación crítico-participativa

lleno de fallas y corrupción. Por consiguiente, su meta se realizó por medio de la reflexión sobre la capacidad de los medios de comunicación para educar a las personas en pro de una ideología. De igual manera, se deseaba hacer énfasis en las ventajas que tiene la unión de personas y cómo éstas tienen la capacidad de igualar poderes.

Por último, la propuesta anterior contuvo elementos de autodeterminación manifestados por los participantes al buscar la lectura crítica de medios de comunicación en sus lectores. Por otro lado, buscó la unión como aspecto fundamental en las luchas contrahegemónicas. Además, existieron elementos de solidaridad en anteponer las injusticias cometidas contra la mayoría antes que la propia seguridad de los miembros de la organización de resistencia.

En el grupo denominado Ryobi se centró la propuesta en la historia inconclusa denominada como Shiawase no Pursuit, cuya problemática principal estaba enfocada en la otredad y los alcances negativos que pueden llevar este fenómeno. Según los estudiantes, la otredad vista desde el racismo es un mal muy establecido en todos los espacios. Ante esto, ejemplifican que dentro de la misma institución a pesar de haber disminuido considerablemente, aún se llevan a cabo situaciones donde algún estudiante es recriminado por su color de piel.

Respecto a este mismo tema, el racismo se ve como un problema que se desea eliminar del todo. Para el grupo de participantes era inconcebible que se siguieran dando esas manifestaciones. En consecuencia, su problemática se enfocaba en realizar un énfasis en el color blanco y negro -ver figura 3- como dos relaciones conflictivas que logran puntos en común para efectuar un cambio en esta perspectiva. 


\section{Figura 3}

\section{Imagen sobre resistencia en "Shiawase no Pursuit"}

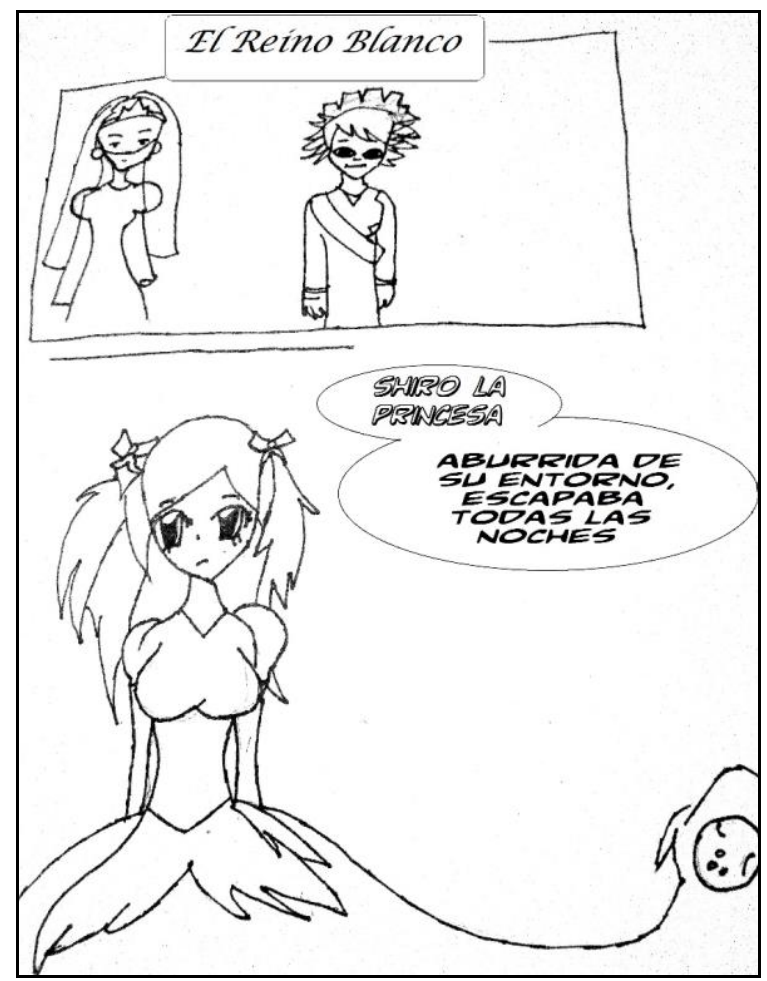

Fuente: Ryobi, Shiawase no Pursuit, diciembre 2015.

Nota: La princesa Shiro muestra señales de resistencia ante las condiciones que le plantean sus padres.

Los alcances de este interés no fueron los esperados debido al abandono del proyecto. Sin embargo, la idea se centraba en mostrar a la población estudiantil los males del racismo, comentar sobre los problemas que representan a la persona agredida y crear conciencia por medio de valores como la igualdad. Mientras tanto, las debilidades de la propuesta estuvieron inmersas dentro de la limitada profundización que se llevó a cabo en el material hecho por los estudiantes, pero sí lo hacía la interpretación al explicarlo al resto de compañeros.

Continuando con Shiawase no Pursuit, a pesar de que algunos estudiantes no se identificaban con la historia, sí se vinculaban con su punto central. Los estudiantes afirmaban que el fin que perseguían era la representación del racismo como problemática 
social desde las diferencias que los etiqueta, aunque los estudiantes no se encuentren en situaciones similares. No obstante, los participantes si asimilaban aspectos dentro la otredad como es el caso de las preferencias sexuales por medio de la intolerancia.

Como punto final, la meta a la que se orientaba la propuesta del grupo Ryobi estuvo manifestada por medio de diseños con rasgos de anime en género demográfico yaoi y yuri, ${ }^{5}$ con lo cual la otredad se representa en aspectos profundizados y de denuncia social usando un estilo que busca la visualización de las preferencias de género. La reflexión del manga está vinculada con la teoría de Walker (2009) que vincula denuncia social y enlaza los aspectos de sacrificio con elementos de esperanza. Los estudiantes tuvieron presentes las ideas de racismo y los aspectos negativos que han producido dentro del país. De igual manera, se da la denuncia para generar reflexión de los males dentro de cualquier contexto que además, se vincula con una trasformación política en la ideología que poseen las personas "diferentes" y que por medio de los lectores hubiesen sido asimilados como iguales.

Concluyendo con las propuestas, se pueden observar que los estudiantes lograron generar manifestaciones de cultura política crítica en sus creaciones. Esto se evidencia en las señales de pensamiento crítico provenientes desde la autodeterminación, codeterminación y solidaridad (Klafki, 1987). Por otro lado, también se presentaron intenciones de acción política transformadora, cuyo propósito les permitiría a sus lectores un cambio de perspectiva sobre los asuntos que detallan en sus obras.

Como se mencionó en el apartado anterior, para la divulgación de los mangas se elaboraron dos presentaciones. Estas consistían en comunicar a las personas el proceso de construcción de las obras, la intención de los autores con los mensajes emitidos y el cambio que esperaban que tuvieran los demás al leer el contenido. La primera de estas dos exposiciones se elaboró en el marco de la feria colegial y la matrícula de algunos grados. Por lo tanto, estuvieron presentes padres de familia y estudiantes de la institución. Por otro

\footnotetext{
${ }^{5}$ Yaoi y Yuri se tratan de series de anime diseñadas para un público meta gay y lésbico respectivamente.
} 
lado, la segunda se realizó durante el ciclo normal de clases y contó con la presencia de trabajadores y alumnos del colegio.

Este primer acercamiento contó con la presencia de participantes identificados con cosplay como se puede observar en la figura 4. La presentación contó con la exhibición de los mangas Kokoro Dream, Spinne Himmel y los bocetos de Shiawase no Pursuit. Esta actividad tuvo la dificultad de contar con un stand lejos de la mayoría de actividades de la feria. Por consiguiente, la cantidad de personas que se acercaron fue limitada respecto a aquellos que se mantenían alrededor. Se esperaba contar con mayor presencia de lectores, ya que este evento posibilitaba el establecimiento de diálogos con personas de otros entornos diferentes al educativo. Esto provocaría que el mensaje de los participantes fuera comunicado a diferentes espacios sociales. De igual manera, se logró conversar con algunos grupos de personas que se acercaron a mirar los mangas y conversar con los participantes.

\section{Figura 4 \\ Fotografía de la primera presentación en las instalaciones del colegio}

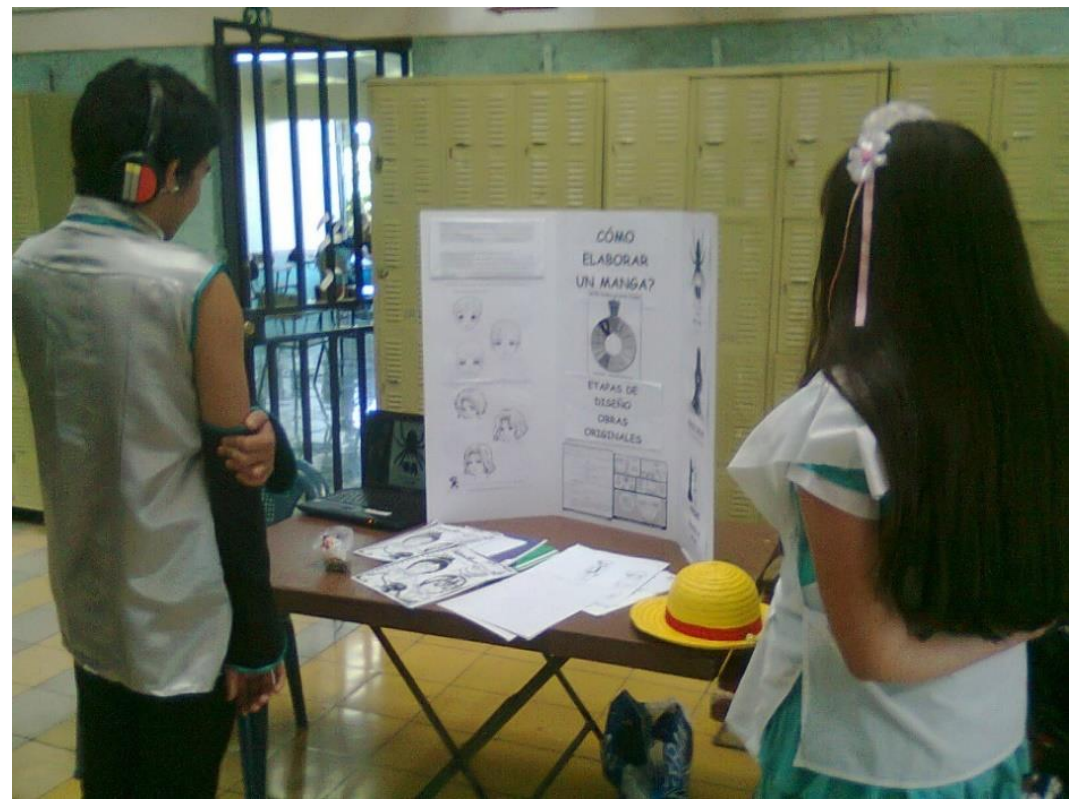

Fuente: Elaboración propia (diciembre, 2015). 
La acción política como herramienta metodológica en implementación de propuestas: un caso de investigación crítico-participativa

Un primer grupo de personas se mostró curiosa al ver que las obras aún no estaban terminadas. Por lo tanto, los estudiantes les hablaron de la composición del proyecto y que este tipo de creaciones llevaban un proceso largo de elaboración, en especial por mantener al mismo tiempo sus actividades académicas. Además, los espectadores visualizaron que estas historietas eran diferentes y tenían un mensaje significativo, aunque interesándose más en el diseño. Finalmente, las descripciones del grupo Wasabi Shippuden fueron atrayentes y concisas para los visitantes, no así las de los estudiantes que componían Ryobi, ya que tuvieron problemas de fluidez en la comunicación y eso provocó dificultades para demostrar la intención de su historia.

El siguiente conjunto de personas tuvo una disputa con los participantes respecto a los símbolos religiosos dentro del anime y manga. Al respecto, un hombre señaló la presencia de la figura del personaje llamado Mr. Satán dentro de la serie Dragon Ball Z. En consecuencia, los estudiantes de Wasabi Shippuden y Sagitario intentaron argumentar que era una representación de una religión ajena a la de los creadores de la obra y que el personaje mencionado era símbolo del fanatismo obsesivo por una figura heroica que realmente era un mentiroso. Este aspecto mostró una concordancia con el sentido que manifiestan los grupos, en especial Wasabi Shippuden y el tema de las obsesiones. Sin embargo, no fue posible crear un consenso con el visitante, el cual se marchó posteriormente.

El grupo final de personas dentro de esta primera exposición tuvo un mayor acercamiento a los diálogos y propósitos de los mangas. En este ámbito, los estudiantes explicaron detalladamente su creación y expusieron el motivo del manga al público. De esta manera, los participantes lograron orientar a los visitantes y desarrollar diálogo crítico, el cual estuvo ligado a situaciones cotidianas ligadas con la narración de las historietas creadas. Por lo tanto, las propuestas fueron evidenciadas en conjunto con el entretenimiento y vinculación de los lectores.

Respecto a la segunda presentación, esta fue realizada con las obras completadas y publicadas en formato de revista, tanto en físico como en digital y cuya portada se puede observar en la figura 5. Por tal propósito fueron usadas las instalaciones del colegio y la 
actividad estuvo orientada hacia los estudiantes no participantes del proceso y los trabajadores de la institución. Como se puede visualizar en la figura 6, este evento contó con computadoras y dos revistas físicas para poder leer los mangas, así como una exhibición sobre el proceso de construcción de las obras, una mesa para conversar con los autores y un stand para solicitar la versión digital de la revista y escribir comentarios.

Figura 5

Imagen de portada de la revista con mangas creados por estudiantes

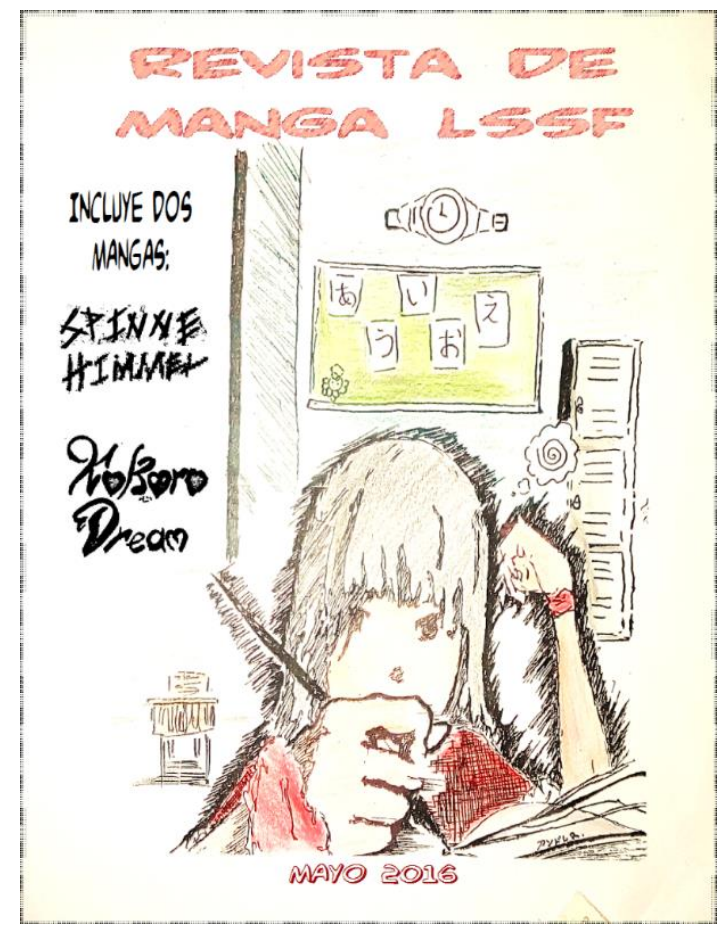

Fuente: elaboración propia (mayo, 2016).

Nota: Portada de la revista que incluye solamente incluye las obras terminadas. 
Figura 6

Fotografía de la segunda presentación

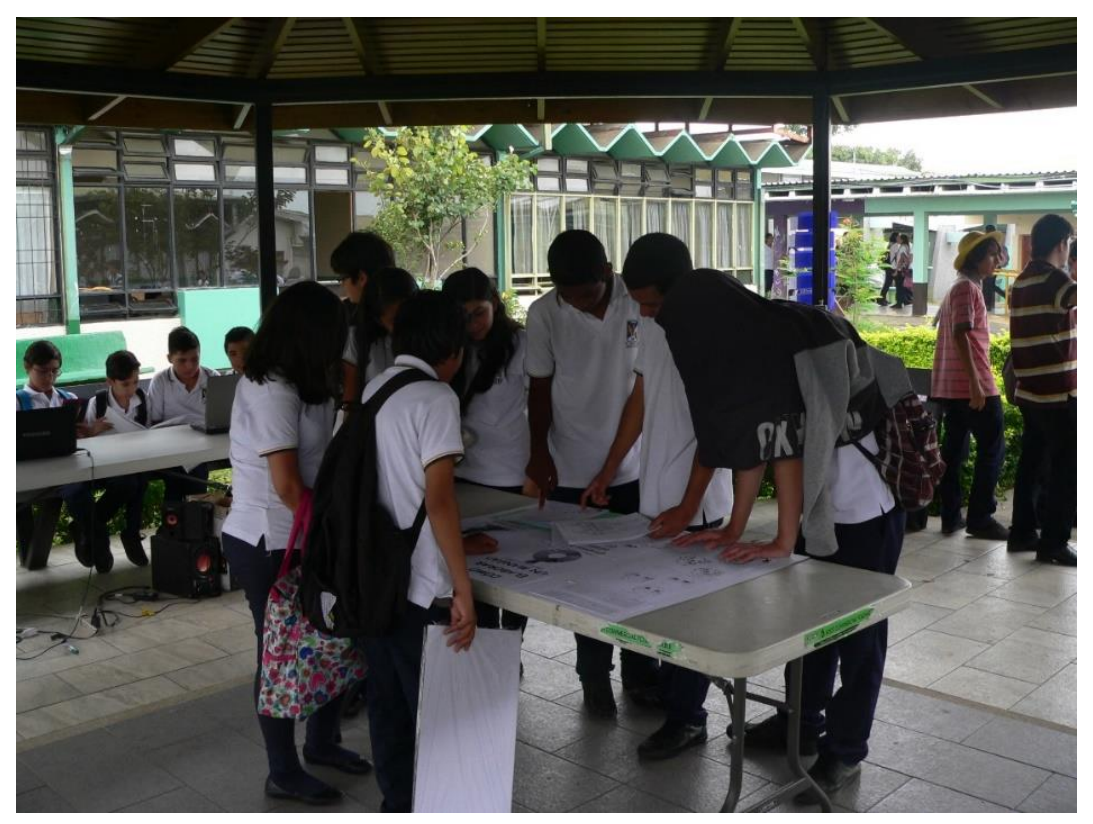

Fuente: Elaboración propia (mayo, 2016).

Nota: En el centro de la imagen se visualiza una exhibición del proceso de creación de los estudiantes que podían ver los mangas tanto en físico como digital.

El detalle que más sobresale de este evento fue la gran cantidad de espectadores con respecto a la primera versión realizada en la feria. Esto se debe a que la segunda actividad tuvo mayor coordinación con la institución y las obras estaban terminadas. Además, el proceso de difusión y convocatoria a la actividad tuvo mayor alcance, lo cual incluyó un espacio exclusivo en el tiempo lectivo, así como la prestación de un lugar visible y de fácil acceso.

Como aspecto general de esta actividad, los visitantes se orientaron más a la parte de dibujo que a las historias. Además, algunos se veían sorprendidos y atraídos por el manga, pero no preguntaban a los autores sino que escribían comentarios como se puede visualizar en la Figura 7. Por otro lado, otros intentaban imponer que sus creaciones eran mejores o aconsejaban a los creadores. Sin embargo, consideraban que las historias eran de 
calidad y que sólo necesitaban retoques, lo cual estaba descontextualizado al no haber formado parte del proyecto y el proceso de los mangas. De igual manera, los creadores no generaron vínculos suficientes para llamar la atención y hablar sobre sus propuestas.

\section{Figura 7}

Fotografía de estudiantes visitando la segunda actividad

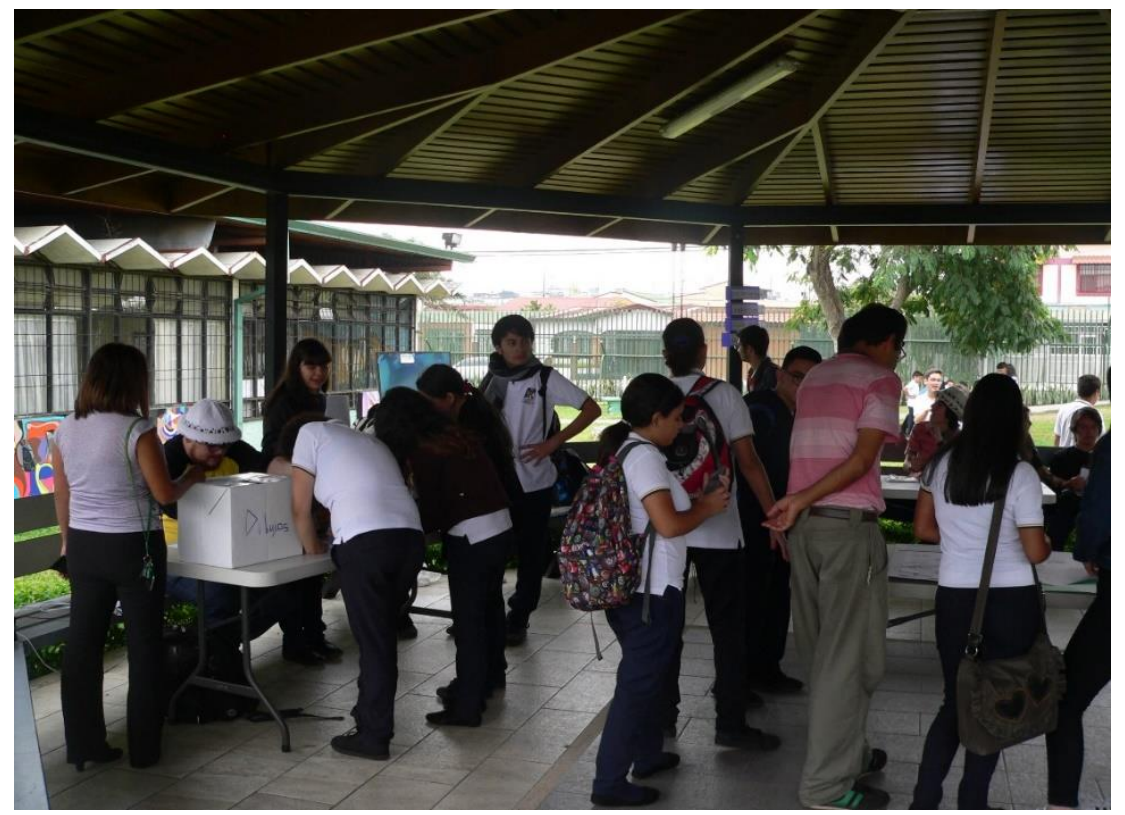

Fuente: Elaboración propia (mayo, 2016).

Nota: La apertura de espacios de expresión de los jóvenes fue un punto importante durante toda la investigación. La segunda actividad tuvo este énfasis, lo cual se ve expuesto en la imagen por medio de un stand para dar comentarios sobre el evento.

En este contexto, el grupo Sagitario tomó mayor determinación respecto a Wasabi Shippuden y tuvieron mayor alcance respecto a su historia Spinne Himmel. Ante esto, los miembros de Sagitario fueron enfáticos en que su obra fue motivo de codeterminación y que hubo un trabajo conjunto al realizarla. Por otro lado, dieron énfasis al proceso que conllevó la finalización del manga, el cual estuvo disputado entre tres historias diferentes. Por consiguiente, los participantes denotaron el uso del diálogo y la llegada a consensos para poder decidir el resultado final de su obra.

Continuando con Sagitario, este grupo también expuso sus intencionalidades al mencionar sobre su manga que: "trata de un grupo de mercenarios que se revelan en contra 
La acción política como herramienta metodológica en implementación de propuestas: un caso de investigación crítico-participativa

de un presidente que parece justo, pero es una muy mala persona que está privando a los demás de sus derechos y entonces el pueblo se revela" (Ana, observación participante 2, mayo 2016). En consecuencia, hubo concordancia con el sentido que manifiestan los autores al no desviarse de la propuesta inicial y dejar en claro sus intenciones.

En otro tema, Wasabi Shippuden se mostró pasivo respecto a la primera actividad. Lo anterior se justifica dado que en el primer caso hubo una intención por exponer la propuesta, la cual se vio opacada por un foro de preguntas y respuestas en el segundo evento. Por lo tanto, los autores trataron de contestar curiosidades como los orígenes, el proceso de construcción, las inspiraciones, el género temático entre otros aspectos de forma. Contrariamente, las intenciones de su propuesta fueron opacadas en esta ocasión.

Como observación general de esta segunda actividad, el propósito original de la desmitificación de las obras fue alcanzado. Lo anterior se justifica debido a la intención de los comentarios dados por los visitantes. Algunas de estas manifestaciones fueron: "me encantan este tipo de actividades, es una linda oportunidad para expresarse y resaltar el arte en el que lo tiene", "me alegra que tengan la oportunidad de expresarse pues es una técnica que pocos conocen", "me parece excelente la motivación y apoyo a los jóvenes; como aprovechar el arte como medio de expresión y desarrollo de aptitudes" (Visitantes de la segunda presentación, observación participante 2, mayo 2016). Lo ejemplificado determina que los asistentes consideraban la creación de mangas como un medio importante para la libertad de expresión y la emisión de intencionalidades.

Continuando el mismo tema, se realizaron comentarios de apoyo por parte de visitantes que no tenían contacto directo con el anime o el manga. El tema principal de los comentarios fue la expresión, lo cual se evidenció en el acercamiento de las personas y las felicitaciones a autores e investigadores. Al mismo tiempo, las críticas se orientaron hacia aspectos como la integración de géneros demográficos como el seinen, ya que el público meta se enfocó exclusivamente en el shonen y shoujo. ${ }^{6}$

\footnotetext{
${ }^{6}$ Las series de anime y los mangas se dividen según su público meta, en este sentido seinen es el género para hombres adultos, shonen para hombres adolescentes y shoujo para mujeres adolescentes.
} 
Concluyendo este segmento, se reafirma la construcción de una cultura política enfatizada en el pensamiento crítico. Esto se manifestó en la construcción de propuestas enfocadas en la solución de problemáticas que consideraban cotidianas y a su vez, referenciadas por elementos observados en el anime. Además, los estudiantes valoraron el papel de las personas más allá de otro tipo de figuras para generar mayor conciencia en los lectores e identificación para denotar la función crítica de sus mangas.

Los estudiantes fueron los principales protagonistas en ambas actividades. Ante esto, se realizaron manifestaciones de solidaridad y codeterminación dentro de la distribución de funciones dentro de cada evento, es decir fueron capaces de llegar a consensos sobre la colaboración de cada uno en la conformación de ambas actividades. De igual manera, siempre se quiso dar autonomía a los participantes para que expusieran sus proyectos y tuvieran el mayor papel dentro del desarrollo del proyecto, ya que los investigadores fueron solamente observadores y mediadores del proceso de construcción de cultura política.

Ambas actividades permitieron romper con mitos orientados en la figura del anime y manga por medio de la consecución de consensos. Primeramente, se trataron de asociar elementos religiosos, los cuales fueron justificados por los autores. Por otro lado, no hubo mención de la violencia como problemática en ninguno de los eventos, al contrario fue expuesta la intención de dar mensajes de cambio a los lectores. En consecuencia, existió concordancia entre las propuestas y los resultados del cambio esperado, siendo visualizado por sólo un caso de disenso y la llegada de acuerdos y mensajes de transformación en el resto de asistentes en ambos casos.

Por último, otro aspecto que se detalló es que aunque los participantes no hayan sido consultados respecto a sus obras, éstos transmitieron a través de sus mangas el mensaje que querían dar. Lo anterior se justifica, ya que las personas se acercaron a leer las obras, en especial Spinne Himmel y Kokoro Dream, las cuales intentaron ser claras con respecto a su propósito de cambio. Esto se vio manifestado en los comentarios realizados por los asistentes que enfatizaban la función del anime y manga como medio educativo y promotor de la libertad de expresión. 


\section{Conclusiones}

Los asistentes de ambas actividades fueron capaces de percibir las condiciones que tienen los animes y mangas como medios generadores de cultura política. Esto se da porque se hizo sólo una referencia a mitos analizados previamente. Al mismo tiempo, en promedio asistieron a ambas actividades más de cien personas. Por consiguiente, se espera que la percepción recibida de estos medios favorezca a la apertura de espacios y el uso de estas series dentro de la educación y otros ámbitos similares.

Se generaron tres intereses relacionados a la manipulación de los medios, la otredad y las obsesiones. Estas posiciones estaban relacionadas con cuestiones que fueron vistas en las series durante el proceso de investigación o bien que permitieron hacer concientización en los lectores y visitantes durante las presentaciones. Por consiguiente, los participantes generaron un interés por cambiar la imagen del anime y manga y situarlo en un espacio de aprendizaje y reflexión.

En el mismo tema, los deseos por hacer una transformación con sus obras se plasmaron en sus propuestas de cambio. Al respecto, la cultura política se construyó a partir del diálogo que existió entre los compañeros de cada grupo para definir sus propósitos, tanto dentro como fuera del espacio de grupo de discusión y que estuvieron mediados por los análisis hechos previamente con las series y los temas debatidos. Lo anterior se vio ilustrado en la historias del manga y en el cómo sus personajes eran actores de cambio o víctimas de situaciones de peligro y, posteriormente, llegaban a la búsqueda conjunta de soluciones contra ese mal.

Como cierre, la acción política fue congruente con las propuestas fabricadas en la mayoría de los casos. Por lo tanto, los estudiantes siempre intentaron resaltar el sentido de su obra como algo diferente y que permite la búsqueda de alternativas ante diferentes hechos. Esto se vio ejemplificado en los diálogos de Wasabi Shippuden durante la primera exposición y de Sagitario en la segunda, los cuales evidenciaban ese deseo por promulgar el mensaje que emiten sus historias manga. Finalmente, se demostró que la apertura de espacios de expresión empodera al estudiante tanto en el arte como en el uso del lenguaje y 
le permite dar sus visiones de mundo y manifestar sus deseos de transformación del entorno.

La aplicación de metodologías de acción política, y en especial aquellas con intención transformadoras, pueden convertirse en posibilidades efectivas para aplicar en otros contextos y con otras temáticas o intenciones. Además, se pudo visualizar que este ejercicio es ideal para poblaciones pequeñas y de uso en investigaciones o proyectos de orden comunal o estudiantil, los cuales pueden derivar en sistematizaciones de experiencias o artículos científicos.

\section{Referencias bibliográficas}

Apple, M. (2008). Ideología y currículo. Madrid, España: Ediciones Akal.

Arendt, H. (1997). Qué es la política. Barcelona, España: Paidós.

Godínez, A., León, E. y Pescod, H. (2016). Anime y Manga como medios de construcción de cultura política: Una experiencia con estudiantes del Liceo Samuel Sáenz Flores. (Tesis de Licenciatura). Universidad Nacional de Costa Rica, Heredia, Costa Rica.

Klafki, W. (1986). Los fundamentos de una didáctica crítico-constructiva. Comunicación: Estudios Venezolanos de Comunicación, 83, 37-79.

Walker, G. (2009). The Filmic Time of Coloniality The Place Promised in Our Early Days. Mechademia, 4, 3-18. DOI: http://dx.doi.org/10.1353/mec.0.0082 